\title{
Recommendation of RILEM TC 218-SFC: Sonic methods for quality control of fresh cementitious materials*
}

\author{
Testing of fresh concrete by ultrasound transmission
}

\author{
RILEM Technical Committee (Hans-Wolf Reinhardt)**
}

(C) RILEM 2011

\section{Introduction}

This recommendation deals with the determination of the stiffening and hardening of fresh cementitious

\footnotetext{
*The text presented hereafter is a draft for general consideration. Comments should be sent within 6 months of publication to the TC chairman, Prof. Hans-Wolf Reinhardt,

Department of Construction Materials, University of Stuttgart, Pfaffenwaldring 4, 70569 Stuttgart, Germany.

Phone: +49-711-68563323; Fax: +49-711-68567681,

Email: reinhardt@iwb.uni-stuttgart.de
}

**This recommendation was developed by a work group within RILEM TC 218-SFC under the leadership of Prof. Hans-Wolf Reinhardt, Germany.

TC-Membership:

Chairman: Hans-Wolf Reinhardt.

Secretary: Christian U. Grosse.

Members: D. Aggelis, Greece; N. De Belie, Belgium; M. Krüger, Germany; M. Ohtsu, Japan; N. Robeyst, Belgium; S.P. Shah, USA; and R. Tatarin, Germany.

Acknowledgement: The contributions by Prof. Bouhadjera, Algeria, and Prof. J.S. Popovics, USA, to the work of the TC are gratefully acknowledged.

RILEM Technical Committee 218-SFC (Hans-Wolf Reinhardt) $\square$

Department of Construction Materials,

University of Stuttgart, Pfaffenwaldring 4,

70569 Stuttgart, Germany

e-mail: reinhardt@iwb.uni-stuttgart.de materials, mainly mortar and concrete. The method uses an ultrasonic pulse which is transmitted through a sample of fresh material. The transit time is measured which leads with the known travelling distance to the velocity of the longitudinal wave (simply called pulse velocity) in the material. The pulse velocity correlates with the elastic properties. By taking measurements on a regular basis during the first hours of hydration the transition from the fresh state to the hardening state can be clearly detected.

\section{Basic principles of the method}

A pulse of longitudinal waves (primary or P-wave) is produced by an electro-acoustical transducer which is held in contact of the wall of a container filled with fresh cementitious material (in the sequel called concrete). After traversing a known path length $L$ in the concrete the P-wave is converted to an electrical signal by a second electro-acoustical transducer which is mounted to the opposite wall of the container. By picking the arrival time of the wave (transit time $t$ ) and after some corrections the wave velocity $v$ is calculated as

$v=L / t(\mathrm{~m} / \mathrm{s})$

If the whole arriving pulse is measured it can also be evaluated with respect to the transmitted energy and the frequency spectrum (see Sects. 7 and 8). By 
repeating measurements, e.g. with a measurement interval of 1-5 min, the method allows for a quasi continuous evaluation of hardening concrete right after mixing.

\section{Testing devices}

\subsection{Principles of container geometry}

The container has two parallel side walls made out of a transparent polymer material (polymethyl methacrylate). A principle is shown in Fig. 1. The thickness has to be at least $10 \mathrm{~mm}$. In the middle of the side wall some recesses allow for the insertion of nylon wear caps in which the sensors (preferably Panametrics type V601-RB or V602-RB) are screwed. The thickness of the plastic caps is app. $1 \mathrm{~mm}$. Between the sensors and the plastic caps some silicone oil has to be inserted to achieve adequate coupling. Between the side walls the bottom part of the container-out of U-shaped closed cell EPDM foam-is clamped in which the fresh concrete can be filled. The foam has to be soft and water-impermeable and should be tested with respect to its acoustic wave propagation properties before being used. The distance between the fixations of the side walls is such that waves possibly propagating through the walls and the fixations after signal excitation do not interfere with

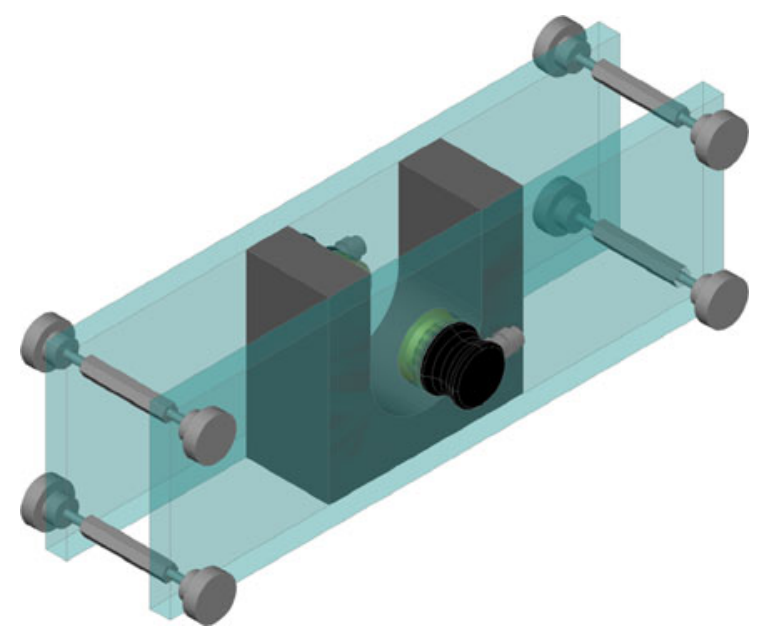

Fig. 13 D sketch of a container for concrete measurements in through-transmission the signals that are directly transmitted through the concrete. The actually needed distance depends on the type of concrete and has to be predefined before testing (see below). A slower mix (e.g. cellular concrete) requires a longer side wall. For a rough estimation of the needed distance between the fixations the following formula could be used:

$l_{\min } \geq \frac{V_{\text {PMMA }}}{V_{\min }} d$

at which $l_{\min }$ is the clear distance between the fixations, $d$ the distance between the two side walls, $v_{\text {PMMA }}$ the pulse velocity of the compressional wave of the side walls and $v_{\text {min }}$ the minimum compressional wave velocity of the concrete that is expected to be measured during the test.

The fixation of the walls has to be demountable for calibration and cleaning. This ensures also that the container is reusable. The width of the U-shaped part can be smaller for investigations of mortar and have to be larger for concrete according to the maximum aggregate size. The minimum width and height of the U-shaped part must ensure test specimen dimensions that exceed the sensor dimensions and sensor area. To ensure proper sealing the width of the compressible foam has to be in minimum the distance between the side walls. The distance between the side walls has to be at least three times the biggest aggregate size of the mixture. An optimization of the container size is recommended to minimize the amount of wasted material as well as to minimize the transit path of the ultrasonic waves along with their attenuation. A larger container can generally also be used to test small grain size materials. However, every change in the test setup and test container may influence the results from the measurements. It has to be noted that small distances between the side walls require high sampling rates to assure required accuracy (see Sect. 6.2.3).

\subsection{Container geometry for cementitious material of not larger than $4 \mathrm{~mm}$ grain size}

Figure 2 represents the required dimensions of a container recommended for materials with small grain size (not larger than $\varnothing 4 \mathrm{~mm}$ ). The distance between the side walls is $22 \mathrm{~mm}$ what results in a volume of about $35 \mathrm{~cm}^{3}$ for the tested material. 

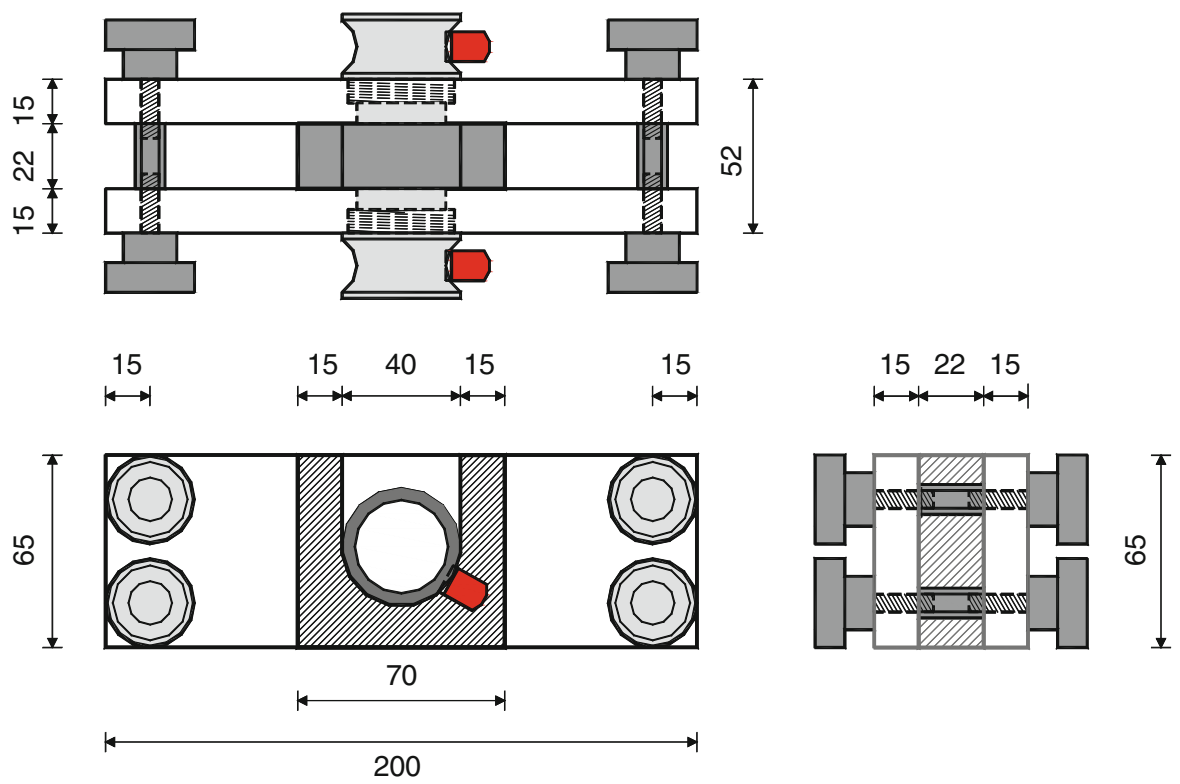

Fig. 2 Mortar container for materials with grain size of not larger than $\varnothing 4 \mathrm{~mm}$ (measures in $\mathrm{mm}$ ) [1]

\subsection{Container geometry for cementitious material} of larger than $4 \mathrm{~mm}$ grain size

Figure 3 represents the required dimensions of a recommended container for materials with larger grain sizes $(\varnothing$ larger than $4 \mathrm{~mm}$ but less than $20 \mathrm{~mm}$ ). The distance between the side walls is $60 \mathrm{~mm}$ what results in a volume of about $450 \mathrm{~cm}^{3}$ for the tested material.
3.4 Container geometry for cementitious material with low pulse velocity

The small and the big containers presented in the last sections were recommended to be used for materials with pulse velocities of not less than app. $250 \mathrm{~m} / \mathrm{s}$. If lower pulse velocities are expected, the distance between the fixations has to be increased according to Eq. 2 and checked by preliminary tests.
Fig. 3 Concrete container for materials with grain size larger than $\varnothing 4 \mathrm{~mm}$ but less than $20 \mathrm{~mm}$ (measures in $\mathrm{mm})$ [1]
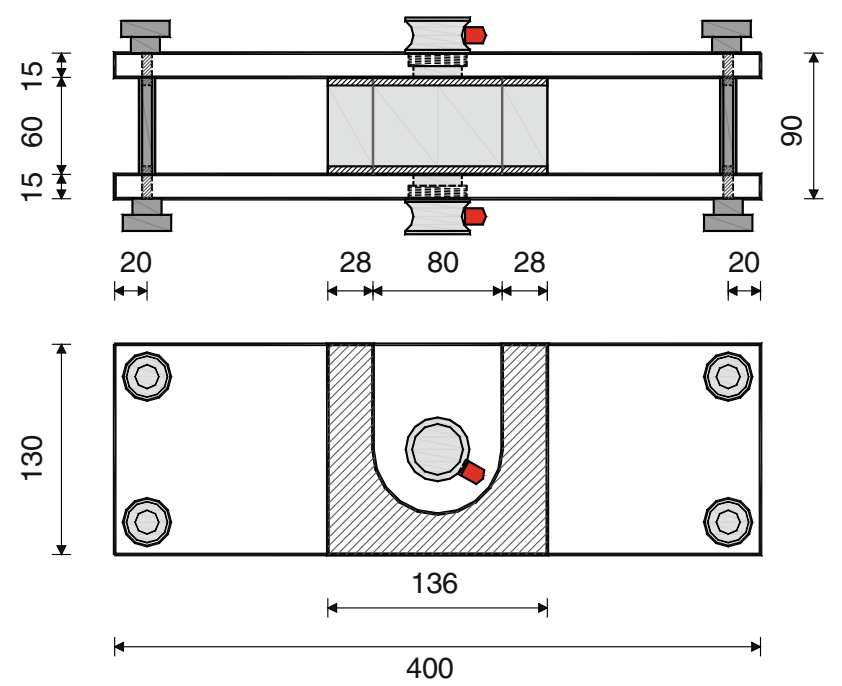
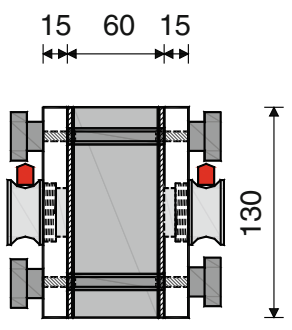


\subsection{Transducers}

The type of sensors that have to be used are sensors that exploit the piezoelectric effect. Their characteristics play an influential role in the recommended measurements (Fig. 4). Since the attenuation of cementitious materials is relatively high right after mixing one would probably try to operate such piezoelectric sensors in resonance, i.e. the signals are recorded within a small frequency range due to the frequency characteristics of the transducer (Fig. 4a). The disadvantage is that an analysis of the frequencies present in the signal is of no value, because these frequencies are always the same. Very well damped sensors, such as those used for vibration analysis, are operated outside of their resonant frequency allowing broadband analyses to be performed, but are usually less sensitive. It is recommended to use broadband displacement sensors that have a flat frequency response (i.e. the sensor gives the same response over a wide frequency range, see Fig. 4c) that are as sensitive as possible. Appropriate broadband sensors are the Panametrics V601-RB with a center frequency of $0.5 \mathrm{MHz}$ and the Panametrics V602-RB with a center frequency of $1.0 \mathrm{MHz}$. If other sensors are used their applicability has to be checked and validated in a separate test series. To enhance the signal-to-noise ratio a power amplifier can be used on the emitter side.

If a characterization of the material under test allows an investigation of pulse velocities only (i.e. no energy or frequency analysis is needed) the use of resonant sensors can be discussed. However, since these sensors are limited in their frequency band the determination of onset times can be inaccurate due to a similar effect as described in Sect. 6.2.3. In this case broadband transducers need to be used. A workaround could be the use of multi-resonant sensors (Fig. 4b) that have a frequency transfer function combining several resonances over a broad frequency range. However, the use of such a sensor type for frequency interpretations is very limited. It is not acceptable to compute and interpret the frequency response of the material during setting with resonant or multi-resonant sensors.
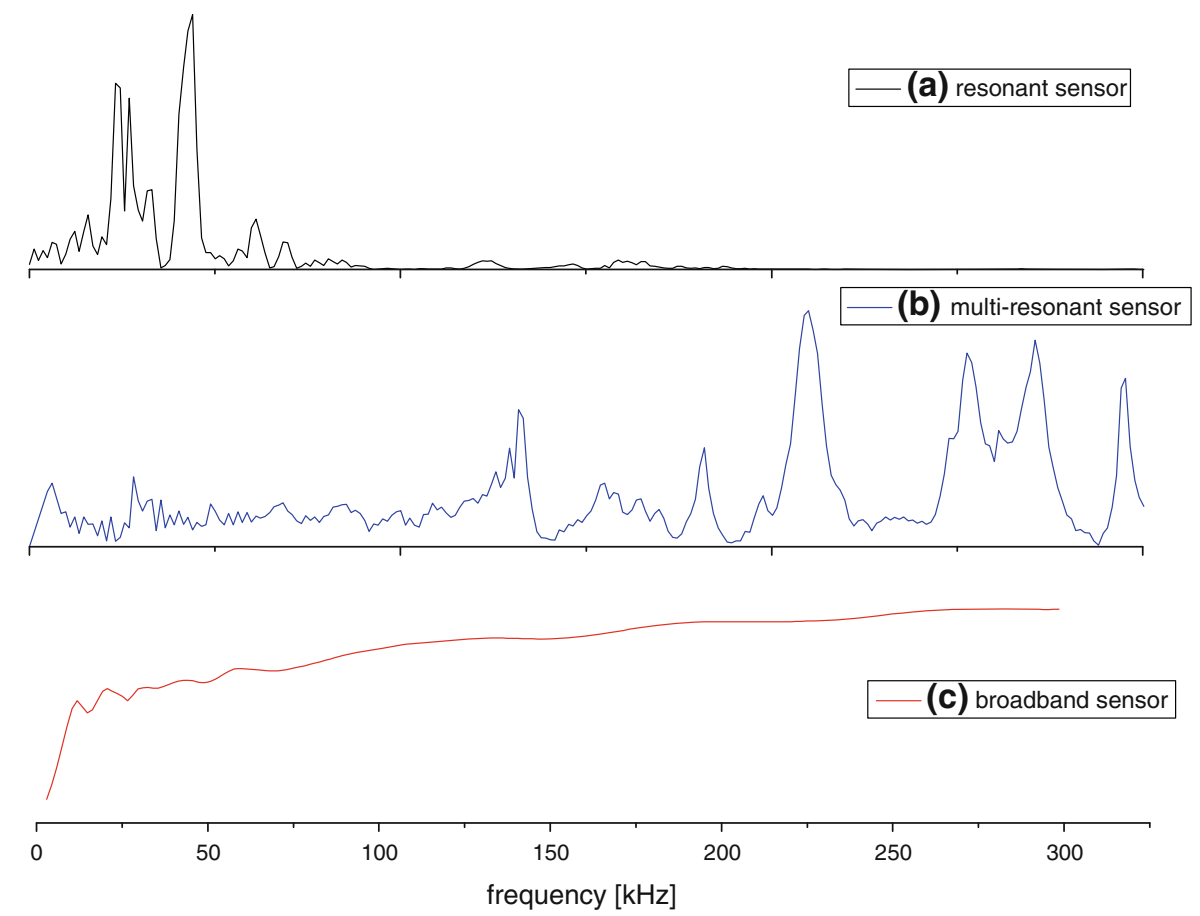

Fig. 4 Frequency transfer functions (sensitivity over frequency) of piezo electric sensors with different characteristics 


\subsection{Description of the measurement device}

A DataAcQuisition (DAQ) system has to be used for logging data and simultaneously as a triggered function generator which enables to record the start of the outgoing signal for example by using a positive slope with amplitude of $4 \mathrm{~V}$ as a trigger pulse (see Sect. 5.2). Figure 5 describes a typical test setup. For most applications the test system consists of a frequency generator, a power amplifier, a measurement container and a preamplifier as well as of an A/D converter and a computer. For the pulse excitation it is recommended to use hardware that could generate rectangular pulses with amplitudes up to $800 \mathrm{~V}$ and a signal length down to $2.5 \mu$ s to excite broadband pulses. Excitation with half sine waves could be used as an alternative, but results on smaller bandwidth of the excited pulses. In most cases it might be good to interconnect a preamplifier between the receiving transducer and the A/D converter to improve the signal quality. In regard to recording signals two parameters are crucial: the sampling interval $\tau$ ( $\mu \mathrm{s}$ ) and the number of samples $n$. The measurement time is then equal to the total recorded time of each ultrasonic signal and is calculated by the product of the two. While the sampling interval should not be larger than $1 \mu$ s (i.e. less data points) it is recommended to record signals with not less than $1 \mathrm{~ms}$ length including the pre-trigger time (see Sect. 5.3). An even shorter sampling interval is recommended if the precision of the onset time determination (see Sect. 6.2.3) is not good enough as can be seen from velocity/time graphs. In these cases a digitizer with at least $5 \mathrm{MHz}$ sampling frequency at 12 bit amplitude resolution would be required.

\section{Sample preparation}

Testing of the setting and hardening of different materials requires reliable conditions with respect to:

- Ambient temperature $\left(20^{\circ} \mathrm{C}\right.$ recommended for standard test procedure)

- Mixture composition and ingredients

- Mixture equipment and mixture procedure

- Filling the container and compaction of the material

- Storage of the container during the testing.

To keep variation in the testing procedure as low as possible it is recommended to follow the standard EN 196-1 [2] for most of the above mentioned aspects, i.e. the arrangements for testing cementitious materials are well defined. Special arrangements could be made with respect to the individual testing objectives. However, such other arrangements should also be traceable, clearly specified and documented.

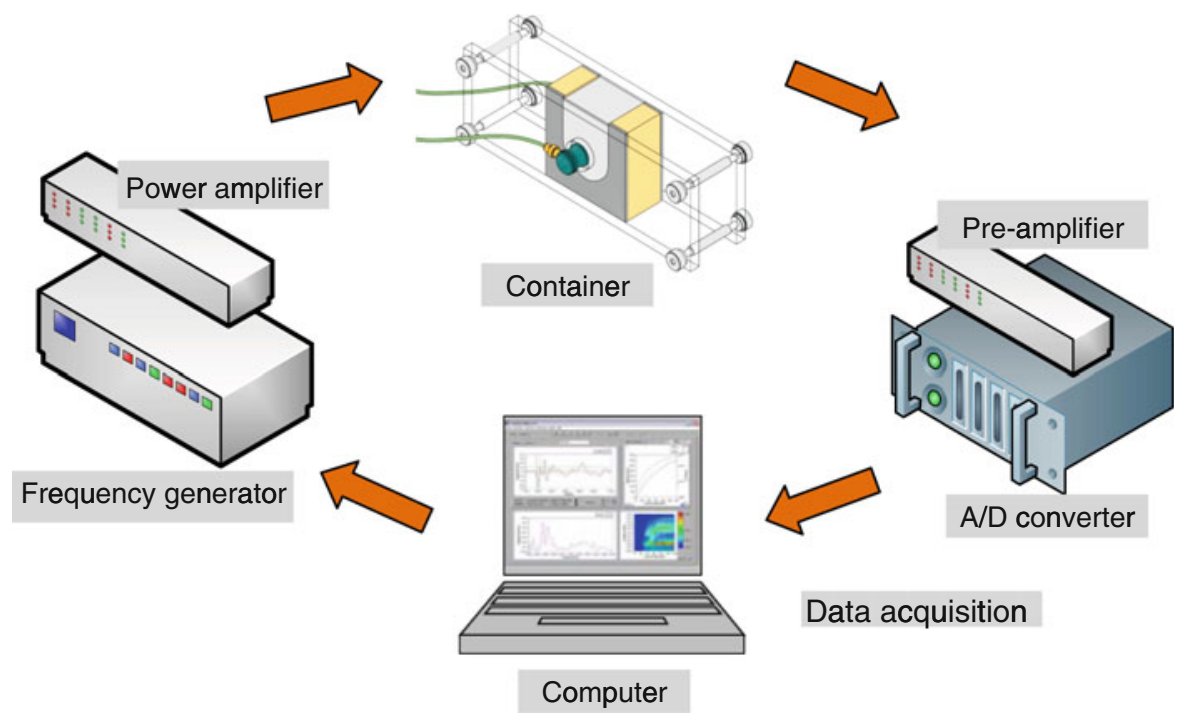

Fig. 5 Typical elements of the test equipment 


\subsection{Mixing the material}

A traceable mixing procedure is needed for all kinds of mixtures to be tested. If the mixture is prepared for the ultrasonic testing only, a minimum amount of 0.51 of material should be mixed. Mixing procedure as described in EN 196-1 is recommended if applicable.

4.2 Filling the container and compaction of the material

The container should be moulded immediately after the preparation of the mix. For stiffer mortar as well as for concrete mixtures that have to be compacted it is recommended to follow the procedures mentioned in EN 196-1. For most of the ultrasonic testing cases the procedure should be the following:

- Fix the container to a jolting apparatus or other reliable compaction equipment similar to that described in EN 196-1 and its Appendix

- Fill the container with the first of two layers of material

- Compact the first layer with the compaction apparatus

- Fill in the second layer of material

- Compact the second layer with the compaction apparatus.

For free-flowing or self compacting mixtures no compaction is needed. However, for filling the container a trough with an adequate length has to

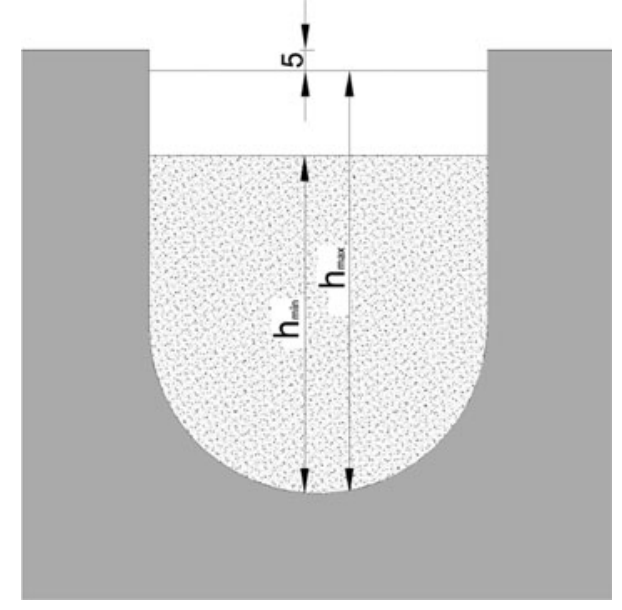

Fig. 6 Filling height be used to allow self-compaction during the flowing process and to achieve homogeneous filling.

The filling height of the container should be kept within the following limits (see Fig. 6):

- For the small container: $h_{\min }=40 \mathrm{~mm} ; h_{\max }=$ $47.5 \mathrm{~mm}$

- For the big container: $h_{\min }=80 \mathrm{~mm} ; h_{\max }=$ $100 \mathrm{~mm}$.

\subsection{Protection of the test specimen against evaporation and specimen storage}

The filling height should be about $5 \mathrm{~mm}$ less than the height of the container. To avoid evaporation and drying out the mix during the test period the specimen should always be sealed with adhesive tape or equivalent material. Covering the specimen right at the beginning of the test with a thin water layer (2-3 mm) might also be effective. Former tests have shown that this does not affect the mixture composition (especially W/C ratio) neither the velocity measurements significantly [1].

The container should be stored on top of an EPDM rubber foam similar to that of the U-shaped form of the container. If ambient temperature conditions are not guaranteed to be constant over the testing period additional temperature measurements are obligatory for validation purposes.

\section{Time signals and determination of the transit time}

\subsection{Ultrasonic time signals}

The signals received in through-transmission have to be recorded and stored for documentation and later verifications. All necessary information about the recording procedure like the sampling rate, pretrigger interval, signal length, amplifications, absolute time of the measurement, filter settings and so far have to be recorded along with the time series.

The signal recordings can be plotted afterwards as shown in Fig. 7. The amplitude of the signals is usually recorded in relative amplitude units, if no absolute calibration is available. Changes of amplitude or energy, respectively, as well as of the frequency content can separately be investigated to 
Fig. 7 Example of changes of the recorded time signals (time after mixing is given above each signal in h:min), amplitudes in relative units

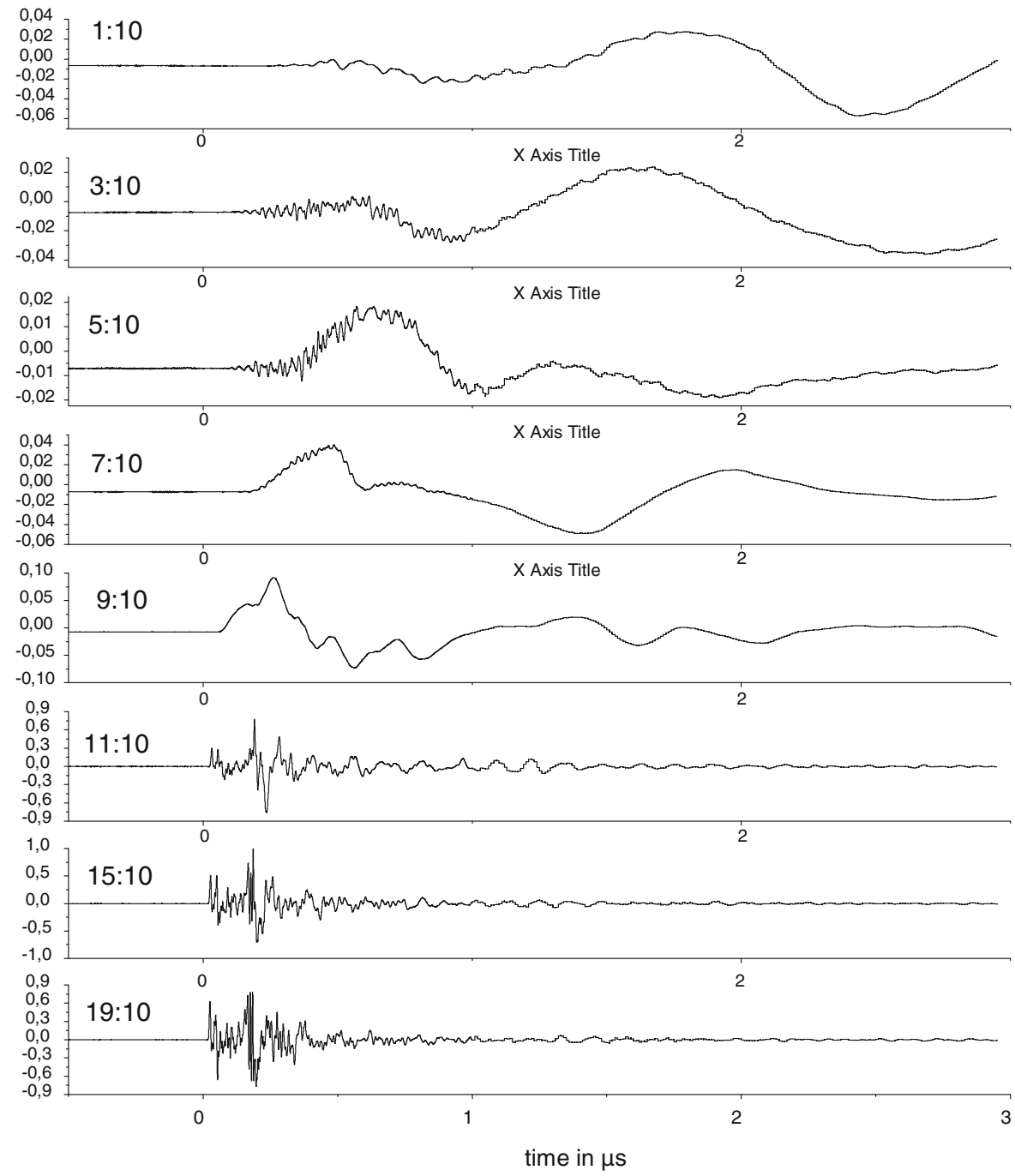

characterize the setting and hardening process (see Sects. 7, 8).

As will be discussed in Sect. 5.3 it is recommended to do repetitive signal recordings in a time window of not more than one second to reduce the statistical error. The average signal usually has a better signal-to-noise ratio by a factor of $\sqrt{n}$, if $n$ is the number of repetitions. The number of repetitions has to be stored along with the data as well.

\subsection{Measurement of the emission time}

The transit time is the time difference between outgoing and incoming signal. The transit time is together with the distance between the side walls the basis for any pulse velocity calculations (see Sect. 6). The outgoing time corresponds to the trigger time of the piezoelectric transmitter sensor. A trigger pulse (e.g. transistor-transistor logic, TTL) is used to start the emission of the pulse-the emitting part of the ultrasound equipment has to be able to produce this TTL signal with an accuracy of better than $1 \mu$ s and to record it. Any delay between the trigger time and the actual emission time of the signal from the first side wall needs to be subtracted according to Sect. 5.4 with at least the same precision as the TTL pulse time. Such a delay can occur due to the time necessary to travel through the container wall and the sensor case. 


\subsection{Measurement of the arrival time}

The transmitted signal is recorded by a sensor attached to the opposite side wall. This signal has to be converted from analogue to digital and to be recorded by a transient recorder. The most crucial part is then to extract as accurate as possible the onset time (i.e. the arrival time) of the signal in relation to the trigger time. If the total recording time $t_{\text {tot }}$ is $1 \mathrm{~ms}$ (see Sect. 3.6) a pre-trigger time interval of at least $15 \%$ (i.e. $150 \mu \mathrm{s}$ ) has to be set to establish for a reliable onset time detection. These first $15 \%$ of the signals can be considered as noise. The onset of the signals has to be discriminated from noise. There are various possibilities to do this since there are, threshold and energy based methods and autoregressive processes. An example how to use the Akaike Information Criterion (AIC) [3] as a detector algorithm is given in the Appendix.

It is recommended to do repetitive measurements of $t_{\mathrm{tot}}$ in a time window of not more than one second to reduce the statistical error (see Sect. 5.4). The resulting $\bar{t}_{\text {tot }}$ value has to be recorded together with the estimated error that consists of a standard deviation and a systematic error. Regarding the error analysis the ones considered by the procedure described in Sect. 5.4 (averaging of $t_{\text {tot }}$ ) as well as the ones due to measurements of $t_{\mathrm{c}}$ (see following section) and $L$ have to be included.

\subsection{Correction of the transit time}

When the total transit time $t_{\text {tot }}$ has been measured the real transit time has to be computed by subtracting the time which is necessary to travel through the container wall and the sensor case $t_{\mathrm{c}}$ (see Sect. 6.2.1; calibration). So the real transit time $t_{\text {tot }}^{\prime}$ is

$t_{\mathrm{tot}}^{\prime}=t_{\mathrm{tot}}-t_{c}(\mathrm{~s})$

$t_{\mathrm{c}}$ has to be determined on the container material according to Sect. 6.2.1. Afterwards the mentioned repetitive measurements of $t_{\text {tot }}$ in a time window of not more than one second to reduce the statistical error have to be done. If $n$ (with $n \geq 3$ ) is the number of these measurements the influence of noise is reduced by this averaging by a factor of $1 / \sqrt{n}$ and then $\bar{t}_{\text {tot }}$ results in:

$$
\bar{t}_{\mathrm{tot}}=\frac{\sum_{n=1}^{n} t_{\mathrm{tot}, n}}{n}(\mathrm{~s})
$$

This results with $t_{\text {tot }}^{\prime}$ from Eq. 2 in

$\bar{t}_{\text {tot }}=\frac{\sum_{n=1}^{n} t_{\mathrm{tot}, n}^{\prime}}{n}=\frac{\sum_{n=1}^{n}\left(t_{\mathrm{tot}, n}-t_{\mathrm{c}}\right)}{n}(\mathrm{~s})$,

where $\bar{t}_{\text {tot }}^{\prime}$ is the sum of the corrected total transit times. For simplification this is further on called $t$ :

$t \equiv \bar{t}_{\text {tot }}(\mathrm{s})$.

In most cases a repetition of three times $(n=3)$ is good enough, but it depends on the type of material under investigation.

\section{Pulse velocity}

\subsection{Calculation of the pulse velocity}

When the average corrected total transit time $t$ has been calculated the pulse velocity $v$ of the compressional wave yields analogue to Eq. 1

$v=L / t(\mathrm{~m} / \mathrm{s})$,

with $L$ the clear distance of the container walls. Since $L$ is subjected to errors this effect has to be taken into account for the error estimation of the total error.

\subsection{Calibration, accuracy and reproducibility} of the pulse velocity

\subsubsection{Calibration of the time/velocity measurement}

As mentioned in Sect. 5.4 the time the signal needs to travel through the sensor case and the walls of the concrete/mortar container have to be measured prior to investigations of setting and hardening. Usually, this calibration time $t_{\mathrm{c}}$ is in the range of several $\mu \mathrm{s}$, but it is necessary to subtract this time, because its influence is increasing during the setting observation reciprocal to the reduction of the travel time. This calibration should be redone from time to time or even prior to each measurement campaign. It is done by removing the U-shaped rubber foam part of the container. Both side walls are pressed together and the time $t_{\mathrm{c}}$ is determined without any material in between. If repetitive measures of $t_{\mathrm{c}}$ show deviations an average value can be calculated similar to $t$. 


\subsubsection{Calibration of the sensors}

The calibration of the sensors used can be done according to international standards. We recommend the phase-to-phase calibration [4] because it is easy to be implemented and can be done with less time and little expenses.

\subsubsection{Accuracy and reproducibility of the velocity calculations}

It also has to be ensured that the distance $L$ is not changed throughout the measurements. Usually, the measurement of $L$ is-in addition to $t$-subjected to errors as well. If that is the case, error propagation algorithms considering $L$ and $t$ have to be used to determine the overall accuracy of $v$. The error calculated in this way represents the reproducibility of the velocity determination.

If in particular during the initial phase of the measurements the calculated velocity values are not changing and/or are abnormally high, it has to be checked, whether the side wall distance is too small resulting in waves travelling around the container instead of through it (see Sect. 3.1).

The transit time gets significantly shorter during the setting process. The determination of onset times is therefore more and more difficult the lower the $t$ value becomes. A sampling interval (see Sect. 3.6) short enough to enable for accurate onset picking is recommended.

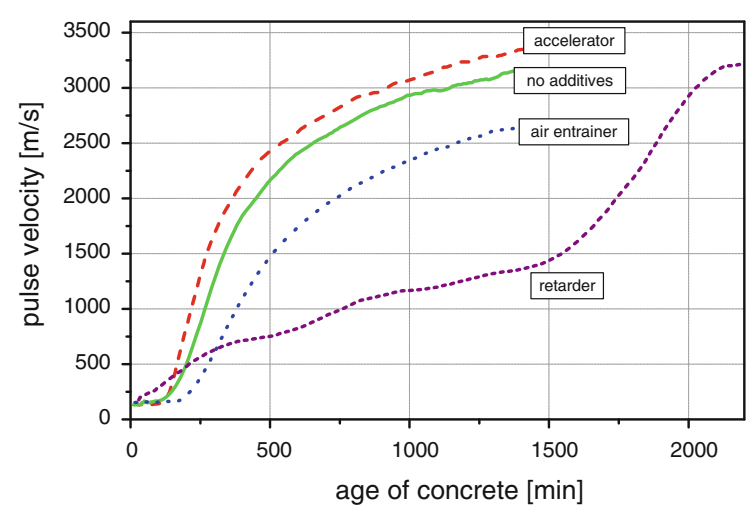

Fig. 8 Example of velocity development over setting time of concrete (age of concrete) depending on the type of additives added to a standard mix (curves are smoothed)
6.3 Presentation of the pulse velocity over setting time

For the presentation of the pulse velocities over setting times a graph showing the evolution of the velocities calculated as described in Sect. 6.1 and based on the onset time data measured as described in Sect. 5 can be plotted. Usually the velocity is a function of the setting time as well as of the material tested - velocity plots of different materials can be combined into one graph. An example for material mixes with different additives added to a standard CEM $42.5 \mathrm{~N}$ is given in Fig. 8.

The curves can be represented in different ways including the single data points or just showing the data curve. It is valid to smooth the plots by using interpolation algorithms as long as the curvature is not significantly changed. In this case it is mandatory to mention the smoothing of the plots. This could in particular be helpful for the higher velocity values where the accuracy is usually lower (see Sect. 6.2.3). Generally, the determined errors should also be represented in the graph.

\section{Transmitted energy ratio}

\subsection{Calculation of the energy ratio}

The transmitted ultrasonic energy $E$ is determined by numerical integration of the squared amplitude values following the onset time.

$E=\Sigma x_{i}^{2} \quad$ with $i=i_{\text {onset } \ldots N}$

$x_{i}$ is the amplitude at sample point $i$ of the signal with $N$ sampling points.

This value is then divided by the reference energy $E_{\text {ref }}$ to eliminate the energy loss due to divergence (beam spreading) and reflection at the contact interfaces (transducer-couplant, couplant-plastic wear cap, plastic wear cap-sample). The reference energy is measured during the calibration of the set-up. The reflection at the plastic-sample interface changes in time as the mortar or concrete sets so it cannot be completely eliminated.

To allow an unambiguous determination of the energy ratio, the following specific settings for the transducer RB-602 were obtained from comprehensive examinations and are preferred to be: 
- Rectangular pulse excitation: Excitation pulse length: $2.5 \mu \mathrm{s}$ (mortar samples), $5 \mu \mathrm{s}$ (concrete samples); Excitation pulse amplitude: at least $400 \mathrm{~V}$ (mortar samples), $750 \mathrm{~V}$ (concrete samples)

- Recording length: $1.638 \mathrm{~ms}(0.164 \mathrm{~ms}$ pretrigger, $1.474 \mathrm{~ms}$ recorded signal).

\subsection{Calibration, accuracy and reproducibility} of the energy ratio

\subsubsection{Calibration of the energy measurement}

The reference energy $E_{\text {ref }}$ is measured with the same set-up and settings as during actual measurements, but with the container filled with water. The average value of at least six measurements of $E_{\text {ref }}$ is calculated.

\subsubsection{Accuracy and reproducibility of the energy calculations}

Shrinkage of the tested specimen can cause the ultrasonic transducers to decouple from the sample. Due to the large impedance mismatch between air and concrete, a small air gap can already cause the energy transmission coefficient to decrease significantly. Due to this large sensitivity of the energy measurements to poor sensor contact, care should be taken to limit drying shrinkage (see Sect. 4.3).

In the period ranging from 4 to $48 \mathrm{~h}$ after mixing, an average repeatability error lower than $7 \%$ can be obtained.

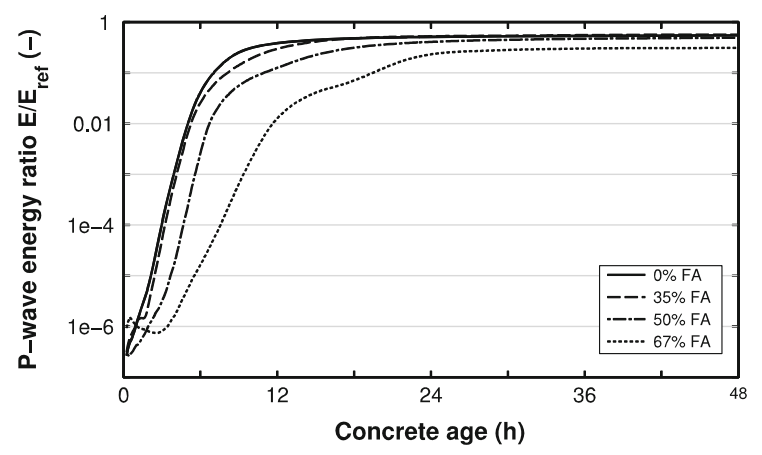

Fig. 9 Example of the change in energy ratio over setting time of concrete for several cement replacement percentages by fly ash (FA)
7.3 Representation of the energy over setting time

Analogously to the velocity measurement, a graph showing the change of the energy calculated as described in Sect. 7.1 can be made (see Fig. 9). The energy is plotted on a logarithmic scale in correspondence with the wave attenuation which is commonly expressed in $\mathrm{dB}$. Generally, smoothing of the energy curves is not required to produce clear graphs.

\subsection{Criteria to determine initial and final setting}

The initial and final setting times according to the penetration resistance test (ASTM C403) [5] can also be indicated by threshold values of the ultrasonic wave energy [6].

For mortar mixtures:

$$
\begin{aligned}
& t_{\text {initial_set }}(\mathrm{h})=t_{E / E_{\text {ref }}=0.02}(\mathrm{~h}) \\
& t_{\text {final_set }}(\mathrm{h})=t_{E / E_{\text {ref }}=0.13}(\mathrm{~h})
\end{aligned}
$$

For concrete mixtures:

$$
\begin{aligned}
& t_{\text {initial_set }}(\mathrm{h})=t_{E / E_{\text {ref }}=0.02}(\mathrm{~h}) \\
& t_{\text {final_set }}(\mathrm{h})=t_{E / E_{\text {ref }}=0.07}(\mathrm{~h})
\end{aligned}
$$

For mixtures with OPC, an average repeatability error lower than $5 \%$ can be obtained for these threshold times.

\section{Frequency spectrum}

\subsection{Calculation of the transmitted frequency spectrum}

The frequency bandwidth of the generated signal depends on the pulse width. The representation in the frequency domain of a rectangular pulse is the sinc function and the width of the first lob (in $\mathrm{Hz}$ ) is the reciprocal of the pulse width (in s). Consequently, a narrow pulse leads to a broader frequency spectrum, but with smaller amplitude at the lower frequencies. Higher frequencies reduce the penetration of the wave but are more sensitive to small discontinuities. Therefore, for concrete samples, which have a larger travelling distance between the sensors, a broader pulse $(5 \mu \mathrm{s})$ is used than for the mortar samples $(2.5 \mu \mathrm{s})$, as mentioned in Sect. 7.1. 


\subsubsection{Filtering}

To eliminate the DC component and low- and highfrequency noise from this signal, a band-pass filter $(100 \mathrm{~Hz}-1 \mathrm{MHz})$ is applied.

\subsubsection{Fast Fourier transform}

The calculation of the fast Fourier transform (FFT) of a transient signal is described in many publications. The relevant issues in relation to the described application are summarized in Appendix.

\subsubsection{Smoothing windows}

During the signal sampling, only a finite record of the signal is obtained. This finite sampling record results in a truncated waveform whose spectral characteristics differ from the original continuous-time signal. The discontinuity at the edges of the signal produces leakage of spectral information, resulting in a discrete-time spectrum that is a smeared version of the original continuous-time spectrum. The amount of spectral leakage increases with the amplitude of the discontinuity. Smoothing windows can be applied to reduce the amplitude of the discontinuities at the boundaries. The time record is then multiplied by a function whose amplitude decreases gradually towards zero at the edges.

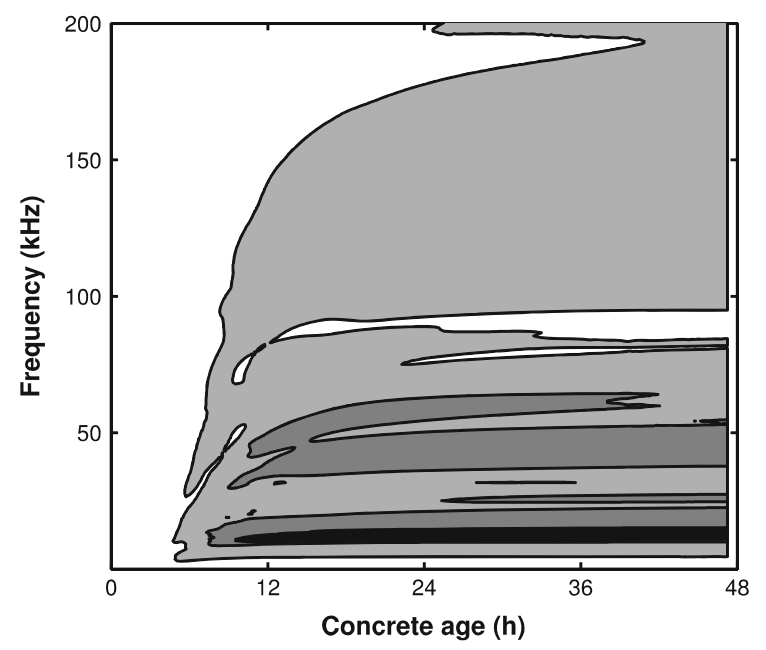

Fig. 10 Contour plots of a fresh concrete sample $(w / c=0.5$, CEM I 52.5): the frequency content is presented by the contour lines in function of the concrete age and the frequency $(-54$, -70 , and $-100 \mathrm{~dB}(\mu \mathrm{V}))$
The received ultrasonic signals are transient signals. When these signals are shorter than the length of the window, no window function should be used. Otherwise, the Hanning window is recommended. The choice of window should not be changed during measurements.

\subsection{Representation of the frequency spectrum over setting time}

The change of the frequency spectrum in time can be visualized in a contour plot with concrete age on the horizontal axis and frequency on the vertical axis (see Fig. 10). Analogously to the energy, the frequency spectrum is plotted on a logarithmic scale expressed in $\mathrm{dB}$.

The first 3-4 h no significant changes are noticed in the spectra, which corresponds with the dormant period of the cement hydration. The high frequencies gradually appear when the concrete starts to set.

\section{Necessary additional measurements}

\subsection{Temperature}

As the ambient and hydration temperature (in the container and in the structural component) essentially influence the measurements, the ambient temperature has to be recorded during the experiment in intervals

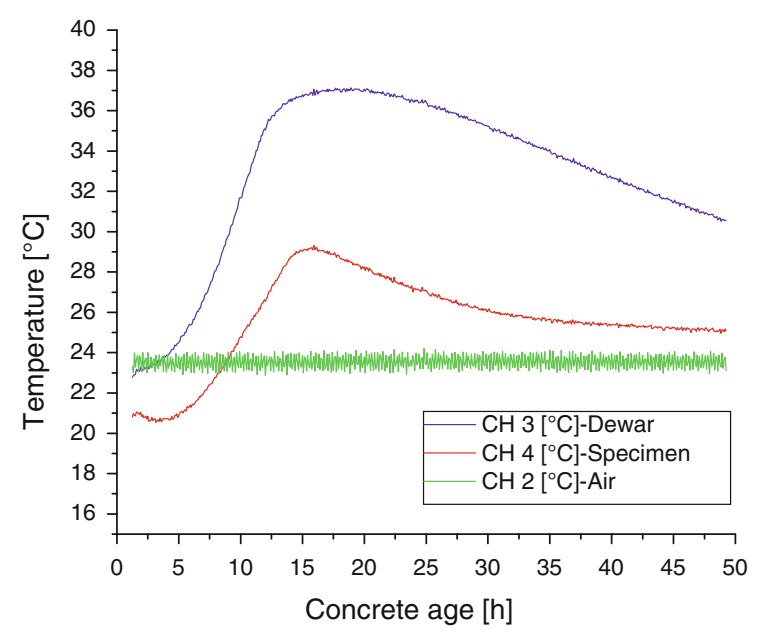

Fig. 11 Example showing the evolution of the hydration temperature in a Dewar container and in the specimen compared to the ambient ("air") temperature 
of an hour or shorter as well as-if possible-the hydration temperature in the container and of a separate sample in an adiabatic or semi-adiabatic container. Parallel measurement of three temperatures is of advantage and can contribute on one hand to increasing the confidence and on the other giving additional information.

The ambient and the hydration temperature in the container can be measured using commercially available thermo-couples. To obtain quasi-adiabatic data, a Dewar container can be used additionally (Fig. 11). Here, account must be taken of the fact that the hydration process in the Dewar container, which simulates a thick structural component, takes place at a much faster rate and can therefore not be directly compared with the sample. This is also the reason why a direct comparison of these data with the ultrasonic measurements is problematic.

\section{Test report}

The following information for each test shall be given:

1. Identification of type of product

2. Ambient temperature

3. Date of manufacture

4. Size of container

5. Settings of the test device (e.g. measurement intervals, excitation pulse characteristics, sampling rate, etc.)

6. Transit time as function of recording time

7. Pulse velocity as function of recording time

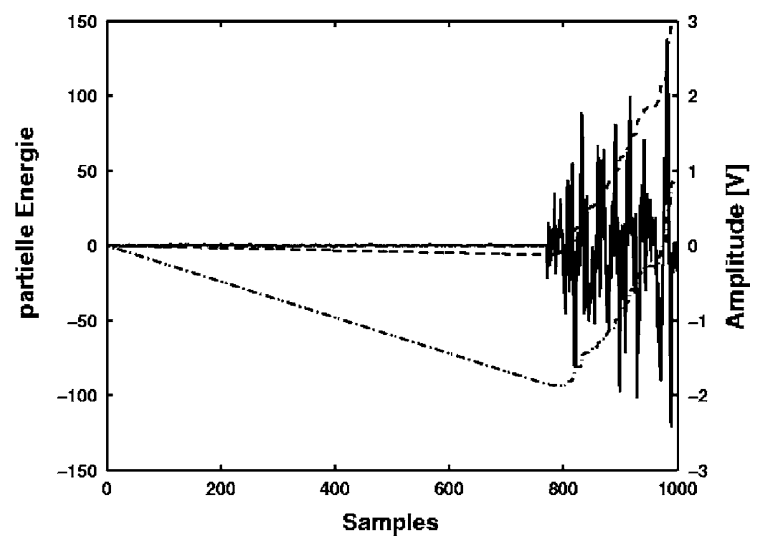

Fig. 12 Example for signal with Hinkley criterion evaluation
8. Transmitted energy as function of recording time

9. Frequency spectrum as function of recording time

10. Special observations
a. air bubbles
b. shrinkage
c. bleeding
d. segregation
e. other observations.

\section{Appendix}

Determination of the onset time

One energy based method uses the Hinkley criterion. The Hinkley criterion can be formulated as follows

$S_{n}^{\prime}=S_{n}-N \delta=\sum_{n=0}^{N} g_{n}^{2}-N \delta$

$g_{n}$ are the amplitudes of all $N$ samples. By introducing a negative trend the function is modified such that the onset time of the signal is represented by the function minimum. The trend is given by

$\delta=\frac{S_{n}}{\alpha N}$

where $S_{N}$ is the total energy of signal length $N . \alpha$ is a correction factor with $0<\alpha<1$. Figure 12 gives an example where the minimum of the lower dashed line represents the onset time of the signal.

Another approach which is based on the autoregressive process is technique based on the AIC (Akaike-Information-Criterion) [3].

The AIC Picker is more complicated to handle because the picking algorithm depends on five parameters, which are further explained in the following.

The picker for the determination of the P-wave onset is based on the Akaike Information Criterion (AIC) [3]. It was adapted to ultrasonic signals by Kurz et al. [7]. The developed autoregressive AICpicker first creates an envelope time function $E(t)$ via the Hilbert transform $\bar{R}(t)$ from the time signal $R(t)$ as 
$\bar{R}(t)=\frac{1}{\pi} \int_{u=-\infty}^{\infty} \frac{R(u)}{t-u} \mathrm{~d} u$

$E(t)=\sqrt{R(t)^{2}+\bar{R}(t)^{2}}$

where $\bar{R}(t)$ is the Hilbert transform; $R(u)$ the time signal; $u$ the current timestep; and $E(t)$ is the envelope time function.

In the envelope time function, a simple threshold level could be used to pick an onset window. The size of the window is specified by the parameters Window upper bound and Window lower bound. These parameters define a number of samples before and after the located time in the envelope time function corresponding to the AIC threshold level.

The AIC function $\operatorname{AIC}\left(t_{\mathrm{W}}\right)$ could then calculated for the selected window as

$$
\begin{aligned}
\operatorname{AIC}\left(t_{\mathrm{W}}\right)= & t_{\mathrm{W}} \log \left(\operatorname{var}\left(R_{\mathrm{W}}\left(t_{\mathrm{W}}, 1\right)\right)\right) \\
& +\left(T_{\mathrm{W}}-t_{\mathrm{W}}-1\right) \log \left(\operatorname{var}\left(R_{\mathrm{W}}\left(1+t_{\mathrm{W}}, T_{\mathrm{W}}\right)\right)\right)
\end{aligned}
$$

where $\mathrm{AIC}\left(t_{\mathrm{W}}\right)$ is the AIC function within the selected window; $t_{\mathrm{W}}$ the time within the selected window; $R_{\mathrm{W}}$ the time signal within the selected window; $T_{\mathrm{W}}$ the maximum time of the selected window; $\operatorname{var}\left(R_{\mathrm{W}}\left(t_{\mathrm{W}}, 1\right)\right)$ the variance of $R_{\mathrm{W}}$ from the current value of $t_{\mathrm{W}}$; and $\operatorname{var}\left(R_{\mathrm{W}}\left(1+t_{\mathrm{W}}, T_{\mathrm{W}}\right)\right)$ is the variance of $R_{\mathrm{W}}$ between $1+t_{\mathrm{W}}$ and the $T_{\mathrm{W}}$.

On the basis of this AIC function it is suggested to cut a second window in which AIC lower bound and AIC upper bound define the number of samples that where cut at the beginning and the end of the AIC function. Within the remaining window the point of the minimum could be determined, which then equals the estimated onset time of the time signal (see Fig. 13). This second cutting of the window is sometimes needed if certain noise (e.g. electromagnetic interference at the very early stage of concrete hardening) lead to multiple minima in the AIC function.

The five parameters of the AIC picking algorithm are to be set individually for different concrete mixes. However, there are some general suggestions that should be taken into account:

- AIC threshold: The AIC threshold should be chosen as low as possible. Values down to 0.001 are feasible.

- Window lower bound: This value should not exceed the amount of pretrigger samples. A good value to start is to set the Window lower bound to the value of the pretrigger samples.

- Window upper bound: This value should be less than the Window lower bound. Preferably Window upper bound should be half of the chosen Window lower bound.

- AIC lower bound: AIC lower bound should be less than Window lower bound for almost all situations. Only in case of very early stage at which the electromagnetic noise dominates the signal and velocity of the tested material is quite low AIC lower bond has temporarily to be set to a value that is 50 to 100 samples larger than Window lower bound.

- AIC upper bound: AIC upper bound must be less than Window upper bound.

Fourier transform

The Fourier transform gives the frequency domain representation of a time signal. Since the received ultrasonic waves are stored by the DAQ card as discrete time signals, the discrete Fourier transform
Fig. 13 Example describing the AIC calculations

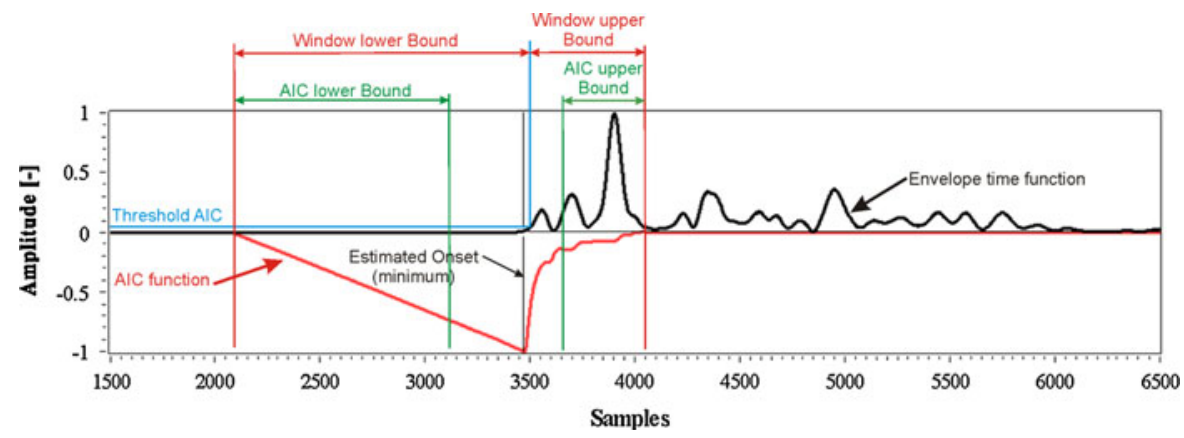


(DFT) is applied. The fast Fourier transform (FFT) is a fast algorithm to calculate the DFT when the number of samples $N$ is a power of 2. If necessary, zeros are added at the end of the signal.

\begin{tabular}{|c|c|c|}
\hline $\begin{array}{l}\text { Time domain } \\
\text { Signal versus time }\end{array}$ & $\begin{array}{l}\text { FFT } \\
\rightarrow\end{array}$ & $\begin{array}{l}\text { Frequency domain } \\
\text { Signal versus frequency }\end{array}$ \\
\hline Sampling interval & & Frequency resolution \\
\hline$\Delta t=1 / f_{\mathrm{s}}$ & & $\Delta f=f_{\mathrm{s}} / N=1 /(N \Delta \mathrm{t})$ \\
\hline Number of samples & & Number of samples \\
\hline$N$ & & $N$ \\
\hline Real signal & & Complex frequency content \\
\hline$x[i]$ & & $\begin{array}{c}X[i] \text { with }|X[i]|=|X[N-i]| \text { and } \\
\text { phase }(X[i])=\operatorname{phase}(X[n-i])\end{array}$ \\
\hline
\end{tabular}

The DFT generates the two-sided spectrum which is symmetrical in the amplitude. Therefore, only the first half of this spectrum contains useful information. Equation 9 shows the computation from a two-sided DFT to a single-sided amplitude spectrum.

$$
\begin{aligned}
X[i] & =\frac{\sqrt{2} \text { Magnitude }[\operatorname{DFT}(x)[i]]}{N} \text { for } i \\
& =1 \text { to } \frac{N}{2}-1 \\
X[i] & =\frac{\text { Magnitude }[\operatorname{DFT}(x)[i]]}{N} \text { for } i=0
\end{aligned}
$$

Multi-way analysis of the received frequency spectra

The dataset with the change of the frequency spectrum in time consists of a 3-way array: the frequency content is measured in function of concrete sample (first mode), frequency (second mode) and concrete age (third mode).

The data should be centered across the second mode, i.e. the frequency mode, to remove possible off-sets. To reduce the dataset, only the frequency band of the spectrum containing the most energy should be selected based on the generated pulse signal (e.g. first $250 \mathrm{kHz}$ for pulse of $5 \mu \mathrm{s}$ ).

\section{Exploratory multi-way analysis}

The most simple and restricted multi-way model is parallel factor analysis (PARAFAC) [8-10] which decomposes a three-dimensional array $\underline{\mathbf{X}}(I \times J \times K)$ into a summation of $R$ trilinear components. Each component is a triple product of vectors, which can be called score vector (sample mode) and loadings (other modes) by analogy with principal component analysis (PCA). A slab $\mathbf{X}_{\mathrm{k}}(I \times J)$ of $\underline{\mathbf{X}}$ can be described by Eq. 16.

$\mathbf{X}_{\mathrm{k}}=\mathbf{B} \cdot \mathbf{D} \cdot \mathbf{A}^{\mathrm{T}}+\mathbf{E}_{\mathrm{k}}$

where $\mathbf{B}$ is the $I \times R$ matrix of loadings, $\mathbf{A}$ the $J \times R$ matrix of loadings, $\mathbf{D}_{\mathrm{k}}$ the $R \times R$ diagonal matrix containing the scores for slab $\mathbf{X}_{\mathrm{k}}$ and $\mathbf{E}_{\mathrm{k}}$ the $I \times J$ matrix of residuals. The main advantage of PARAFAC is the uniqueness of the solution.

If the peaks in the spectrum measurements shift from sample to sample, the trilinearity condition is not fulfilled and PARAFAC will give degenerate solutions with unstable and unreliable parameters. A modified version of PARAFAC known as PARAFAC2 can model data with such shifts in one mode. Unlike PARAFAC, the latter model does not assume parallel proportional profiles from sample to sample in the first mode and is therefore described by Eq. 17 . $\mathbf{X}_{\mathrm{k}}=\mathbf{B}_{\mathrm{k}} \cdot \mathbf{D} \cdot \mathbf{A}^{\mathrm{T}}+\mathbf{E}_{\mathrm{k}} \quad$ with $\mathbf{B}_{\mathrm{k}} \cdot \mathbf{B}_{\mathrm{k}}^{\mathrm{T}}$ constant over $\mathrm{k}$

which is identical to the PARAFAC model in Eq. 16, except that $\mathbf{B}_{\mathrm{k}}$ is not fixed for all the slabs $\mathbf{X}_{\mathrm{k}}$.

To select the optimum number of PARAFAC2 components $R$, the explained variance can be calculated for an increasing number of components according to Eq. 18.

explained variance $(\%)=100\left(1-\Sigma_{i j} e_{i j}^{2} / \Sigma_{i j} d_{i j}^{2}\right)$

In Eq. $18 d_{i j}$ is an element in the raw data and $e_{i j}$ is the corresponding element in the residuals after the modelled variation is removed. The explained variance indicates how well the model approximates to the raw data. The optimum $R$ is achieved if the explained variance does not increase significantly if more components are added.

For each model, also the explained variance after cross-validation should be calculated which can be seen as a measure for the ability to predict new data. Samples can be successively left out and the models build upon these reduced datasets are used to predict the left out elements and calculate the cross-validated explained variance. The optimum $R$ is achieved if the 
cross-validated explained variance stagnates or decreases if more components are added.

Classification of concrete mixtures is achieved through visualization of the samples in score plots which are the projection of the data onto the PARAFAC components. For instance, the scores of the second, third and fourth component are plotted in function of those of the first component. The location of the samples in the score plots are then determined by the setting process. The distances between the scores of two samples indicate how much the change in frequency spectrum from these samples differs.

\section{Basics of multi-way regression analysis}

Multi-way analysis can be performed to relate the change in the ultrasonic frequency spectra, compiled in the predictor three-dimensional array $\underline{\mathbf{X}}(I \times J$ $\times K$ ), to other measured quantities such as the setting times, compiled in the response matrix $\mathbf{Y}(I \times L)$.

Multi-way partial least square (N-PLS) regression, finds a linear regression model by projecting $\underline{\mathbf{X}}$ and $\mathbf{Y}$ to a new space. Analogously to PARAFAC, $\underline{\overline{\mathbf{X}}}$ and $\mathbf{Y}$ are decomposed into $R$ components, which are also called latent variables (LV's). They contain all the relevant information, while redundant data and noise are left in the residuals.

To select the optimum $R$, the errors between the predicted and the actual values can be calculated in several ways. The root-mean-square error of calibration (RMSEC) for a variable is generally defined by Eq. 19.

$$
\mathrm{RMSEC}=\sqrt{ }\left(\Sigma_{i}\left(y_{i}^{\prime}-y_{i}\right)^{2} / N\right)
$$

where $y_{i}^{\prime}$ is the value of the predicted variable when all samples are included in the model formulation, $N$ the number of $y_{i}$ values. The RMSEC is a measure of how well the model fits the data.

The model's ability to predict new samples is investigated by performing a cross-validation. The calculation is analogous, except $y_{i}^{\prime}$ is the prediction for sample $i$ if sample $i$ is not included in the model formulation. Two methods can be used to crossvalidate the model. In the leave-one-out (loo) method, every sample is successively excluded. The model constructed by this reduced dataset is then used to predict the excluded element. In the split-half method, the dataset is split into two parts: a calibration and a validation set. The model constructed by the calibration set is then used to predict the validation set. The root-mean-square error of cross-validation (RMSECV) for a variable is generally defined by Eq. 20.

$\mathrm{RMSECV}=\sqrt{ }\left(\sum_{i}\left(y_{i, \mathrm{cv}}^{\prime}-y_{i}\right)^{2} / N\right)$

where $y_{i, \mathrm{cv}}^{\prime}$ is the value of the predicted variable with sample $i$ not included in the model formulation. Additionally, also the coefficient of determination $R^{2}$ can be used to indicate the accuracy of the prediction.

The optimum number of components $R$ is achieved if the prediction errors increase or stagnate if more components are added.

\section{Example of multi-way regression analysis}

An example of the change of the received frequency spectra in time is shown in Fig. 14a for 3 out of 34 concrete samples studied by Robeyst [1]. Initially, when the concrete is still a fluid-like suspension, lower frequencies of only lower amplitudes can be detected, while the high frequencies are completely damped. The high frequencies gradually appear when the concrete starts to stiffen. The frequency spectrum broadens fastest for the Portland cement concrete without additives (Fig. 14a, \#2) and slowest for concrete with blast-furnace cement type III/C (Fig. 14a,\#12) or with high amounts of BFS or FA added separately (Fig. 14a, \#23). This change of the frequency bandwidth in time can be compared with the evolution of the velocity, described in earlier publications.

To quantify the differences between the spectra of the samples, multi-way analysis was performed as described in the following.

The dataset consists of a three-dimensional array: the frequency content was measured in function of concrete sample (first mode), frequency (second mode) and concrete age (third mode). Several multi-way models were tried out, but the best results were achieved with PARAFAC2. This model is an extension of the more common PARAFAC model which decomposes a three-dimensional array into a summation of triple products of vectors (called components) such that the greatest variance of the data is captured by the first component [11]. Each component is the product of a score vector and two loading vectors. The loading vectors can be seen as the 
Fig. 14 a Contour plots of the frequency content versus concrete age and frequency. b PARAFAC2 Score plots for concrete samples with blast-furnace cements (filled square), blast-furnace slag addition (filled triangle) and fly ash addition (filled circle). The colour indicates the BFS or FA content from black (no BFS or FA) to white (high content)
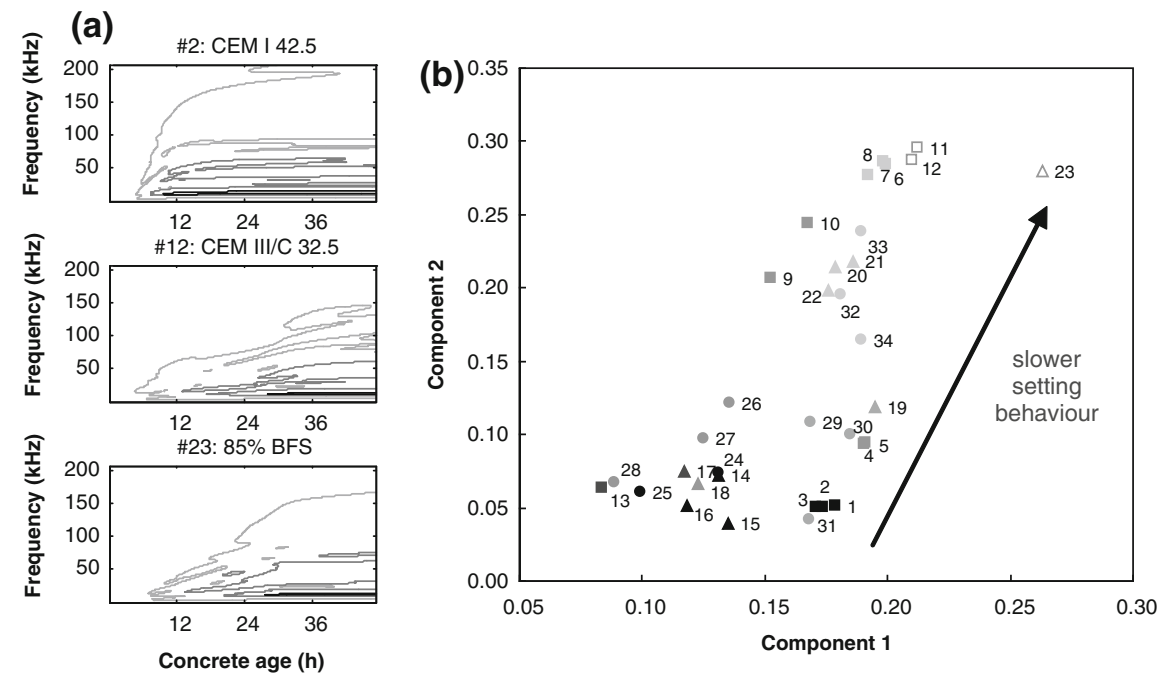

\section{References}

common structures in respectively the frequency and concrete age dimension for the different samples, while the elements of the score vector indicate how much these common structures are present in a certain sample. Classification of concrete mixtures can thus be achieved through visualization of the samples in score plots. The scores of the second component are plotted in function of those of the first component (Fig. 14b). Replicates (e.g. \#1, 2 and 3) are located together, which indicates the correctness of the model. The location of the different samples in the score plots seems to be determined by the setting process:

- the setting decelerates with increasing blast-furnace slag content added to mixtures with OPC: e.g. \# 16 (0\%), 18 (30\%), 19 (50\%), 20 (70\%), 23 (85\% BFS)

- the setting decelerates with increasing fly ash content added to mixtures with OPC:

- e.g. \#24 (0\%), 26 (35\%), 29 (50\%), 32 (67\% FA)

- the setting decelerates with increasing blast-furnace slag content in the blast-furnace cement: e.g. \#1 (CEM I 42.5), 4 (CEM III/A 42.5), 6 (CEM III/ B 42.5).

The scores of the second component and to a lesser degree of the first component seem to increase with slower setting behaviour. Some samples deviate from these general trends, which is discussed in more detail in other publications $[1,12]$. It can be concluded that the sample scores are related to the setting behaviour of the corresponding concrete mixture.
1. Robeyst N (2008) Monitoring setting and microstructure development in fresh concrete with the ultrasonic throughtransmission method. PhD thesis, University of Ghent, Belgium

2. EN 196-1. Methods of testing cement-part 1: determination of strength; German version EN 196-1:2005

3. Akaike H (1974) A new look at the statistical model identification. IEEE Trans Autom Control 19(6):716-723

4. ASTM E976-10. Standard guide for determining the reproducibility of $\mathrm{AE}$ sensor response

5. ASTM C403/C403 M-08. Standard test method for time of setting of concrete mixtures by penetration resistance

6. Robeyst N, Grosse CU, De Belie N (2009) Measuring the change in ultrasonic p-wave energy transmitted in fresh mortar with additives to monitor the setting. Cem Concr Res 39(10):868-875

7. Kurz JH, Grosse C, Reinhardt HW (2005) Strategies for reliable automatic onset time picking of acoustic emissions and of ultrasound signals in concrete. Ultrasonics 43:538546

8. Smilde A, Bro R, Geladi P (2004) Multi-way analysisapplications in the chemical sciences. Wiley, Chichester

9. Bro R (1997) PARAFAC. Tutorial and applications. Chemom Intell Lab Syst 38(2):149-171

10. Wise BM, Gallagher NB, Bro R, Shaver JM (2005) PLS_Toolbox version 3.5 for use with MATLAB ${ }^{\mathrm{TM}}$ (manual). Eigenvector Research Inc, Manson

11. Ebrahimi D, Li J, Hibbert DB (2007) Classification of weathered petroleum oils by multi-way analysis of gas chromatography-mass spectrometry data using PARAFAC2 parallel factor analysis. J Chromatogr A 1166(1-2): 163-170

12. Robeyst N, Grosse C, De Belie N (2009) Monitoring fresh concrete by ultrasonic transmission measurements: exploratory multi-way analysis of the spectral information. Chemom Intell Lab Syst 95:64-73 